\title{
La extensión universitaria en una universidad pública
}

\section{University extension in a public university}

DOI: http://dx.doi.org/10.17981/cultedusoc.11.2.2020.14

Recibido: 11 de marzo de 2020 Aceptado: 25 de junio de 2020 Publicado: 12 de julio de 2020

Guillermo Tenorio Bohórquez

Universidad Internacional Iberoamericana. Campeche (México)

guitebo@yahoo.com

Para citar este artículo:

Bohórquez, G. (2020). La Extensión Universitaria en una Universidad Pública. Cultura, Educación y Sociedad, 11(2). 221-236. DOI: http://dx.doi.org/10.17981/cultedusoc.11.2.2020.14

\section{Resumen}

El artículo analiza el movimiento extensionista y la función extensión ofertada y ejecutada por una universidad pública colombiana del orden nacional, considerando el Artículo 120 de la Ley 30 de 1992 de educación y el Acuerdo 037 de 2004 de los estatutos de la entidad. Con base en una investigación de tipo mixta, se aborda el movimiento extensionista desde referencias de trabajos de diferentes autores de nivel mundial y con encuestasconsulta realizadas a la comunidad académica administrativa de esta universidad, aplicando un instrumento estadístico para poblaciones finitas, con respecto a competencias cognitivas en Extensión Universitaria. Una vez sistematizada la información se identificó el nivel de conocimiento sobre el tema, conllevando a multiplicidad de enfoques y evidenciando la diversidad de criterios respecto a actividades de extensión en esta institución que se manifiestan en la oferta de cursos cuyo perfil no corresponde como tal a la tercera función misional. Se sugiere a la institución objeto de estudio ajustar sus actividades de extensión de acuerdo con la ley de la educación colombiana para la tercera función misional. Palabras clave: Extensión universitaria; proyección social; tercera función misional

\begin{abstract}
The article analyzes the extension movement and the extension function offered and executed by a Colombian public university of the national order, considering Article 120 of Education Law 30 of 1992 and Agreement 037 of 2004 of the entity's statutes. Based on a mixed type of research, the extension movement is approached from references of works by different authors worldwide and with consultation-surveys carried out with the administrative academic community of this university, applying a statistical instrument for finite populations, with respect to cognitive skills in University Extension. Once the information was systematized, the level of knowledge on the subject was identified, leading to a multiplicity of approaches and evidencing the diversity of criteria regarding extension activities in this institution that are manifested in the offer of courses whose profile does not correspond as such to the third missionary function. It is suggested to the institution under study to adjust its extension activities in accordance with the Colombian education law for the third missionary function.
\end{abstract}

Keywords: University extension; social outreach; third missionary function 


\section{INTRODUCCIÓN}

Las condiciones prospectivas para las diferentes sociedades, así como las exigencias de sus desarrollos a todo nivel, demandan relaciones dinámicas entre las IES y las funciones misionales, direccionadas en nuevos modelos sociales con acciones reciprocas en referencia a la interrelación universidad-sociedad, como principio orientador e integrador de la docencia, investigación y extensión con una visión humanista para la educación superior. En otros términos, según Morales, Mira \& Arias (2010), la concepción de Extensión en la universidad moderna emerge en la universidad de Berlín en 1810, para reflexionar en la parametrizacion de la enseñanza y el conocimiento científico, visualizando así la base de las funciones misionales de docencia e investigación. En Serna (2007), se afirma que el concepto de Extensión toma fuerza en América Latina a inicios del siglo XX y tiene como principio rector la justicia social, por cuanto se entiende como obligación de compartir la cultura y los conocimientos con los menos favorecidos. Esta concepción marca una diferencia entre la universidad europea y latinoamericana, porque el cambio social no es el criterio central de las universidades anglosajonas.

La concepción de proyección social hace mención a aquellos servicios y actividades que permiten lograr una mejora en la vida de la gente. El uso más habitual de este modelo se vincula a la educación superior, que se presta para que las personas tengan las herramientas necesarias para progresar socialmente. La proyección social básicamente corresponde a la responsabilidad social que parte de las relaciones empresa-sociedad, como centro en la teoría y justificación de la empresa desde los comienzos del capitalismo, tanto en el debate público como en la reflexión teóricoacadémica. El carácter social de las organizaciones productivas es reconocido por las teorías económicas más antiguas (García-Perdiguero, 2003; Lizcano y Moneva, 2003).

En Latinoamérica, se consolida la Extensión Universitaria con la Reforma de Córdoba, en 1918, fortaleciendo la investigación y la expansión del conocimiento con planteamientos políticos y sociales. Para este estudio en el ámbito colombiano, y teniendo en cuenta que la universidad analizada hace parte de las dieciséis universidades públicas del orden nacional colombianas, catalogadas en el Observatorio de la Universidad Colombiana, se encontró que el cincuenta por ciento de estas universidades en términos de Extensión, optaron por un denominaciones tales como: Gestión Universitaria y Extensión, Investigación y Extensión, Proyección Social y Extensión y Extensión Universitaria, en donde se destaca como mayor exponente la Universidad Nacional de Colombia, que adoptó como tercera función misional la Extensión Universitaria, y con la cual ha obtenido logros en gestión académica y social en términos de Extensión. Por otra parte, el otro cincuenta por ciento de las universidades públicas del orden nacional, optaron como tercera función misional la Proyección Social. Se puede observar que no hay un criterio unificado en este sector de IES con respecto a la tercera función misional. 


\section{Metodología}

Para evidenciar el concepto de Extensión o Extensión Universitaria, se hizo necesario indagar históricamente desde sus inicios, para luego contrastarla con el conocimiento actual que presenta una universidad pública del orden nacional, considerando la metodología de investigación mixta, buscando respuestas al planteamiento central de la investigación. Por ende se hizo recurrente la indagación sobre aspectos de tipo teórico, mediante un mapeo general respecto al movimiento extensionista desde sus inicios. En segunda instancia, se condujo la investigación a la Universidad Colegio Mayor de Cundinamarca-Unicolmayor, que es una institución pública del orden nacional, dado el tipo de actividades que realiza con Extensión como Proyección Social.

Como se indicó anteriormente, se determinó la combinación de los enfoques cualitativo y cuantitativo, es decir, una investigación de tipo mixta, que incluye las características de cada uno de estos enfoques (Grinnell citado por Hernández, Fernández y Baptista, 2003), además señalando que en estos enfoques se utilizan fases similares y están relacionadas entre sí. Por tanto, ese indago el tema de las actividades realizadas con Extensión por la oficina de Proyección Social de esta Institución, mediante trabajo de campo con encuesta-consulta a la comunidad académico-administrativa, los entrevistados se constituyen en una unidad de análisis secundaria no incluidos en el estudio, para no afectar con sus opiniones en el análisis situacional referencial sobre el funcionamiento y vida de la Institución.

Se elaboró un formato de encuesta-consulta, el cual fue aplicado y sistematizado, como instrumento estadístico para poblaciones finitas, para obtener una muestra representativa de la población académico-administrativa de Unicolmayor con respecto al conocimiento sobre Extensión Universitaria como tercera función misional. Para esta investigación, la muestra estudiada "corresponde a un caso aplicable y al contexto real de las instituciones" (Martínez, 2006, p. 55).

Las encuestas personalizadas son totalmente independientes y corresponden al uso de instrumentos ad hoc, que no están relacionadas con ninguna otra encuesta; ello requirió de un diseño específico del formato, para la aplicación de este tipo de instrumento. A fin de sistematizar la información, se consideró una escala de 1 a 5 , para determinar el nivel de conocimiento de la población, con respecto al tema de Extensión o Extensión Universitaria, en donde si el NC es: $1=$ Es muy poco, $2=$ Es poco, $3=$ Es regular, $4=$ Es bueno y $5=$ Es muy bueno.

Sistematizada la información, se elaboró un gráfico de barras como muestra visual de los datos obtenidos en las encuestas, donde se obtuvo un grupo de valores de acuerdo con la escala propuesta a fin de cuantificar y determinar el nivel de conocimiento de la población académico-administrativa de Unicolmayor respecto al tema de Extensión y de la gestión de la Oficina de Proyección Social. Por último la información fue analizada. 


\section{FUnDAMENTACIÓN TEÓRICA}

En 1810, la Universidad de Berlín considerada moderna en su concepción presentó un modelo de enseñanza para la producción del conocimiento, demostrando cómo se había generado; es en este momento en que germina la concepción de las funciones sustantivas de docencia e investigación. Con posterioridad, la función de Extensión es reconocida como tercera función en las universidades con características de impulso y facilitación en doble vía de los conocimientos compartidos entre universidad y sociedad como núcleo central del desarrollo de la educación.

En los anales de la educación pública inglesa, quedó por sentado que el primer programa de Extensión en la Universidad de Cambridge fue en 1867; SE inicia una actividad que posteriormente fue instrumentada por numerosas universidades como la Universidad de Oxford y otras del Reino Unido, con la finalidad de acercar la institución a la población. Cuatro años después, emergió la expresión de Extensión Universitaria Educativa, buscando que los estudiantes universitarios se acoplaran con los obreros y de esta forma crear vías de más fácil acceso para alcanzar a la población más menesterosa en términos sociales. En 1872, en Cambridge se considera la aparición de la Extensión Universitaria, reconocida por la influencia de las diversas reformas y la diversidad de características regionales, bajo esas condiciones las universidades se ubican destacando los principios generales de esta función misional. La función de Extensión Universitaria responde así a un esquema integro de la comunidad con la universidad en calidad de columna vertebral en su ser y saber hacer (Labrandero y Santander, 1987).

Desde esta perspectiva histórica se considera que las instituciones universitarias británicas trabajaron especialmente en la temática de ciencia como tal, entre los siglos XIX y XX, factor que redundó en la merma de posibilidades de las clases populares las cuales fueron tenidas en cuenta solamente a mediados del siglo XIX. Pero realmente, se debe acreditar la labor de los británicos a nivel mundial, quienes dieron consentimiento para que la educación universitaria fuese posible a las clases populares de numerosos países. Según Rábade (1996), se afirma que el inicio de siglo XX marca un hito en la educación superior del viejo continente, por cuanto el modelo educativo alemán ya había dominado la mayoría de las instituciones universitarias de alto prestigio alrededor del mundo y multiplicado con el continente europeo, con una corriente educativa que se caracterizó por la pluralidad de sectores y grupos sociales. Así, se arraizó la función de Extensión Universitaria, con las apodadas Universidades Populares, sin importar las diferencias de los modelos impuestos en Francia, Bélgica e Italia, con sus principios y líneas programáticas.

A finales del siglo XIX, España no escapa de esta evolución, y con el estímulo por parte de las universidades de Zaragoza, Barcelona, Sevilla y Oviedo, para el proceso de la educación popular en la pluralidad de varios sectores sociales. Por ende, la concepción de Extensión Universitaria de la Península Ibérica, mantuvo los principios concebidos en Inglaterra. Posada (1911) afirma: "Toda labor expansiva de carácter educativo y social, realizada por la Universidad fuera de su esfera oficial docente" (p. 73). Se considera que esta es la primera definición de Extensión Universitaria. 
En el nuevo continente a mediados de 1860, en los albores de Extensión Universitaria en Estados Unidos, se hace asequible la educación universitaria a la mayor parte de la población. Khan (1989) indica que en 1862 se aprobó la primera Ley Morrill, para 1890 que autorizaron créditos directos adicionados para las universidades de concesión de tierras para la agricultura, dando origen a los Land Grant Colleges , cuyo principal objetivo respondía a promover la un agro industrializado y factor de progreso tecnológico. El modelo de Extensión Universitaria se activó y se puso en práctica muy rápidamente en ese país, tan solo en 1879, se iniciaron cursos y conferencias fuera de las aulas que permitieron constituir numerosos centros de Extensión Universitaria.

La Extensión Universitaria, se posicionó en Estados Unidos para la formación técnica, acompañada de la experimentación, considerando la extensión para el área rural, en el área agrícola se enfatizó la investigación y la producción específicamente para el sector rural. Los centros de extensión contribuyeron sustancialmente a la modernización, crecimiento y desarrollo productivo de la agricultura norteamericana. En Rosenberg (1982), se indica que este prototipo de universidad extensionista sería base posterior de los hechos reales sobre Extensión Universitaria, en inobservancia de las universidades de corte liberal inclinadas a las artes, que no incluían prácticas requeridas para el agro. Finalizando el siglo XIX e inicio del siglo XX, se continuó impulsando la formulación de programas y a través de creación de unidades denominadas departamentos de extensión en esas universidades. Considerando el caso de la Universidad de Chicago en 1900, consolidando la facultad de Extensión Universitaria, base de las denominadas universidades industriales.

Ferrero (1999) indicó que las universidades latinoamericanas no consideraban ningún tipo relación en ambos sentidos con la comunidad; estaban absortas en la formación de profesionales enclaustrados dentro de las aulas, sin contemplar la posibilidad de extender su gestión más allá de los muros y comunicación a través de actos culturales y grupos reducidos de profesores y estudiantes de la misma elite. En 1918, se celebró el Primer Encuentro Internacional de Estudiantes Latinoamericanos, proclamando la Reforma Universitaria de Córdoba, con el clamor a las IES respecto a su misión social con el entorno, remarcando la necesidad que esta se vincule con la comunidad, bajo la bandera de la educación inclusiva a toda la sociedad, debatiendo sobre la distribución del saber de la educación superior articuladamente de la universidad y sociedad, denotando la responsabilidad que tienen las IES de compartir cultura y el conocimiento con su entorno.

Según Ravelo (2018), la Reforma de Córdoba, que culminó con la propuesta estudiantil denominada El Manifiesto Liminar, permite discurrir académicamente al conminar la clase media por acceder al derecho de la educación superior que anteriormente no les fue concedida. Puede decirse que fue el momento propicio para establecer los programas de Extensión Universitaria. Esa reforma, insto las posibilidades de educación superior para la clase media latinoamericana, basada en una propuesta mucho más que academicista, manifestando voluntades de carácter político y social, llevando entonces a la trasformación estructural de las universidades; considerando que ya no eran las clases privilegiadas las que podían acceder a la educación superior, y finalmente ya estaba el camino abierto para la clase media. 
El primer Congreso de Universidades Latinoamericanas en Guatemala (1949), se ratificó la Función de Extensión, el carácter social de la universidad en lo educativo, social y cultural. Los años cincuenta conceptualizan la Extensión Universitaria, mediante un esquema sistémico, coherente con su función integradora. Y en desacuerdo con ese tipo de IES que trasiegan unidireccionalmente, con visón egocéntrico queriendo salvaguardar el saber y la cultura en calidad de recurso exclusivo, considerándose como única vía para la población, en desmedro del conocimiento empírico y popular. Por ello se aprobó la creación de la Unión de Universidades de América Latina (UDUAL). Latinoamérica, en Chile 1957, que realizó la Primera Conferencia Latinoamericana de Extensión Universitaria y Difusión Cultural, adoptó la Extensión Universitaria (UDUAL, 1957).

Ahora bien, hay que entender que la Extensión Universitaria es como un proceso integracionista de universidad-comunidad y de ésta con la universidad, por cuanto solamente hasta 1972 fue tenida en cuenta, pudiéndose determinar el impacto social de la universidad en la sociedad con la relación entre las partes, con una concepción de contribución a la transformación y perfeccionamiento (Segunda Conferencia Latinoamericana de Extensión Universitaria y Difusión Cultural, UDUAL, 1972). Tünnermann (2000) afirma: "Extensión Universitaria es la interacción entre universidad y los demás componentes del cuerpo social, a través de la cual ésta asume y cumple su compromiso de participación en el proceso social de creación de la cultura y de liberación y transformación radical de la comunidad nacional" (p. 188).

Considerando la Extensión, Tünnermann (2000) relata: "Por su naturaleza, la extensión universitaria es misión y función orientadora de la universidad contemporánea, entendida como ejercicio de la vocación universitaria. Por su contenido y procedimiento, la extensión universitaria se funda en el conjunto de estudios y actividades filosóficas, científicas, artísticas y técnicas, mediante el cual se auscultan, exploran y recogen del medio social, nacional y universal, los problemas, datos y valores culturales que existen en todos los grupos sociales" (p. 217).

En tal sentido, la fundamentación de la Extensión Universitaria en Latinoamérica ha presentado consideraciones y debates en el deber ser y quehacer de la educación superior actual, en escenarios tales como la Conferencia Regional sobre Políticas y Estrategias para la Transformación de la Educación Superior en América Latina y el Caribe en La Habana, Cuba, en 1996 y la Conferencia Mundial sobre la Educación Superior, en París, que señala la necesidad de una universidad moderna involucrada de forma interdisciplinaria con las necesidades de la comunidad y por ende con el medio ambiente.

De igual manera y respecto a la funciones misionales, Verdesoto (1967) afirma: "La Reforma de Córdoba incorpora la Extensión Universitaria y difusión cultural entre las tareas normales de la universidad latinoamericana y propugnó por hacer de ella el centro por excelencia para el estudio científico y objetivo de los grandes problemas nacionales"(p. 7). Con base en el concepto de la Reforma de Córdoba, Tünnermann (2003) considera que "las universidades son instituciones sociales que corresponden a partes del cuerpo social y la extensión es una de sus funciones" (p. 274).

Al compilar modelos de Extensión Universitaria en distintas épocas, catalogándola como altruista, divulgativa, concientizadora, vinculatoria empresarial y otros. Serna 
(2004) afirma: "El concepto de Extensión, que toma fuerza en Latinoamérica a inicios del siglo XX, teniendo como principio rector la justicia social, con obligación de compartir la cultura y conocimientos con los menos favorecidos. Esta concepción marca una diferencia entre universidades europeas y latinoamericanas, considerando que cambio social no es criterio central de las universidades anglosajonas" (p. 2).

En contraposición, otros autores consideran que "este vínculo debe entenderse como un mecanismo que ayuda a elevar la calidad de la investigación y de la docencia, y lograr una mejor integración con las necesidades sociales" (Colmenares, 2001; Misas, 2004; Martínez, Mavarez, Rojas, Rodríguez \& Carvallo, 2006). En tanto que, definitivamente, el enfoque social sobre las prácticas actuales de Extensión Universitaria conduzca a repensar que la academia en todos sus niveles se articule con la comunidad, en sus vivencias cotidianas, desde un plano más real recibiendo y transmitiendo conocimientos que conlleven a la solución de problemas a nivel sociocultural y porque no de tipo laboral (Carlevaro, 2009).

En el siglo XX y albores del XXI, en Latinoamérica se han presentado variedad de modelos de Extensión Universitaria de conformidad con las necesidades propias de los gobiernos. No es secreto que en las IES en la actualidad presentan programas académicos con una tendencia a suplir las necesidades que requieren la oferta y demanda del mercado laboral. En países considerados en vía de desarrollo o tercermundistas, se acentúa ese tipo de preparación profesional, conllevando a la triada de universidad, comunidad y empresa, donde la Extensión Universitaria, como medio para prácticas empresariales de los futuros profesionales.

Posteriormente, en el XIV Congreso Latinoamericano de Extensión Universitaria (ULEU) del 2017 y segundo Congreso Centroamericano de Compromiso Social (CSUCA), celebrado en Nicaragua en la ciudad de Managua, conmemoró un siglo de la Reforma de Córdoba, entre los principales puntos se propuso declarar la Extensión Universitaria como base para el procedimiento educativo, en función de la población para el crecimiento y desarrollo socioeconómico. Asimismo, la Extensión Universitaria en calidad de integradora del conocimiento, abanderada de la investigación y la cultura, llegando a toda la población con ecuanimidad social. Considerando el axioma propuesto en la Reforma de Córdoba, para la modernización de la institucionalidad universitaria.

Por cuanto la labor realizada por el movimiento extensionista ha sido ardua, se han realizado investigaciones, trabajos y ponencias, en los diferentes eventos realizados en Cuba, Argentina, Costa Rica, Venezuela, México, Chile, Brasil, Colombia y Francia. Cabe destacar la III Conferencia Regional de Educación Superior, realizada en 2018, la cual consolido el compromiso de cientos de actores de educación superior ante el presente y el porvenir de la educación superior de la región. En Colombia se constituyó La Red de Extensión Universitaria de la Asociación Colombiana de Universidades (ASCUN).

Ahora bien, frente al paradigma entre Extensión Universitaria y Proyección Social, se debe considerar la Responsabilidad Social Corporativa (RSC), que está orientada a la obligación moral y ética de las empresas en concomitancia con empleados, la economía y otros espacios sociales. Considerando el concepto de Responsabilidad Social empresarial (RSE) en 1953, se iniciaron los estudios con respecto a este tema. Bowen (1953) afirma: 
"todas las acciones y decisiones tomadas por los empresarios impactan directamente la calidad y personalidad del resto de la sociedad. La RSE se refiere a las obligaciones de los empresarios aplicando políticas, tomando decisiones y seguir líneas de acción deseables en términos de los objetivos y valores de la sociedad, y el empresario debe actuar para servir a la sociedad teniendo como objetivo la maximización de los beneficios y como único fin la empresa" (p. 6).

Por ende, la concepción de Responsabilidad Social Universitaria (RSU), intuida en la Responsabilidad Social Empresarial (RSE), concibe el contexto por el cual la comunidad demanda versatilidad empresarial, direccionada a problemas sociales, por ello se consideró que las universidades son organizaciones (empresariales), que tienen impacto en las comunidades y por tanto les compete la responsabilidad social. Todo esto encausado en un esquema de inversión social, conlleva al gasto extra para la empresa con responsabilidad social, por ello que se consideró que la institución universitaria debería tratar de superarlo este modelo. Vallaeys (2006) afirma: "La proyección social y la extensión universitaria como suplementos bien intencionados a su función central de la docencia y producción de conocimientos, para poder asumir la verdadera exigencia de la Responsabilidad Social Universitaria."

En tal sentido, para el caso colombiano la Ley 30 (1992, Art. 120), reconoce que la proyección social es una función misional de las IES, cuyo objetivo es cumplir con su misión social a través de la interacción de la academia con los diferentes contextos, aportando alternativas de solución a problemas y necesidades de los diferentes grupos, contribuyendo así en la transformación de la sociedad. Como entidades autónomas, las IES pueden definir sus acciones amparadas en la autonomía universitaria.

Es conveniente considerar que el proyecto de educación superior en Colombia se inicia en 1580, la reforma educativa se dio a partir del Decreto Ley 80 (1980, Art. 21), organizando de forma estructurada la educación. Además, se erigió el Sistema de Educación Superior, el Sistema Nacional de Ciencia y Tecnología e Innovación (Ley 1286, 2009, Art. 16), para las IES y programas de alto nivel en la educación universitaria.

Debe señalarse que la Extensión Universitaria en el contexto colombiano se realizó con un discurso en el Congreso de la República en 1909, donde se propuso una reforma en la educación superior. Dicho discurso del general Uribe afirma: "Una universidad científica, moderna, experimental, actual y evolutiva”, como postura del Partido Liberal. La Reforma no fue aprobada en 1911 por oposición del Partido Conservador; la propuesta consideró la cátedra libre y Extensión Universitaria como función social, el proyecto fue reiniciado con la reforma de Alfonso Pumarejo de 1935. La Universidad Nacional de Colombia, se convirtió en abanderada en este tema, incorporando la integración de universidad y sociedad para el periodo de 1944 a 1948.

Con una perspectiva más puntual, la Universidad Nacional de Colombia (UNAL, 2009) afirma: "una función misional y sustantiva de la Universidad, a través de la cual se establece una interacción privilegiada y recíproca entre el conocimiento sistemático de la academia y los saberes y necesidades de la sociedad, y de las organizaciones e instituciones que hacen parte de ella" (p. 44).

Dentro de este marco, la Universidad Nacional de Colombia (UNAL, 2009), también considera: "Esta relación entre la Universidad y su entorno se debe reflejar en la 
ampliación del espacio de deliberación democrática y en el bienestar de las comunidades. Con la Extensión se cualifican la ciencia, la tecnología, el arte y la cultura" (p. 44).

De allí, luego de interminables polémicas académicas e institucionales de la Universidad Nacional, se propuso participar en la cimentación del país, creando el Instituto de Estudios Políticos y de Relaciones Internacionales, el Centro de Estudios Sociales y reactivado el Centro de Investigaciones, para procesos de investigación de la población colombiana.

Si bien es cierto, la Institución busco afianzar los programas académicos, dada la falta de una política de Extensión. La función de extensión fue contemplada por el Decreto 80 de 1980 de educación superior en Colombia, para el desarrollo de esta función misional. Posteriormente con la Ley 30 (1992, art. 120), se reorganizó la educación superior en el país que dio pie al concepto de Extensión.

En otras palabras, en el país se consideró la idea de Extensión Solidaria, buscando diferenciarla del modelo extensionista financiero. La Universidad Nacional de Colombia (UNAM, 2009) la ratifica: "como aquella que comprende los programas y proyectos científicos, tecnológicos, artístico y culturales de alto impacto social, que se desarrollan y financian total o parcialmente con recursos de la Universidad".

Se puede señalar la Extensión Solidaria acorde al desarrollo histórico del concepto. Ardila (2011) considera que "desafortunadamente en el país se ha venido usando con fines económicos más que filantrópicos o emancipadores, debiendo la universidad salir al mercado ofreciendo servicios de extensión como una mercancía a través de publicidad" (p. 45).

En este orden de ideas, se hace necesario reflexionar sobre los factores que influyen en los procesos de Extensión Universitaria, aparentemente es sencillo, pero verdaderamente complejo en sus especificidades y todas latitudes. En el Acuerdo por lo Superior 2034, con un plan prospectivo de gestión a corto, mediano y largo plazo, el Consejo Nacional de Educación Superior-CESU (2014) articula la paz y la convivencia como elementos viables para un país educado. Por consiguiente la concepción de la Extensión no concuerda con la de nivel mundial, dado que no existe una política pública de Extensión Universitaria en Colombia, teniendo en cuenta que hasta el momento es considerado tema de estudio en algunas organizaciones, como la Asociación Colombiana de Universidades (ASCUN) aglutinando universidades con Red de Extensión Universitaria.

Cabe indicar que la globalización se deja sentir en la universidad occidental del siglo XXI, hay que repensar en el desgastado concepto del claustro universitario. En tal sentido, "las funciones sustantivas de la Educación Superior, a saber: docencia, investigación, y extensión, requieren hoy, de formas que les permitan crear vínculos que faciliten su integración no solo con campos disciplinares y profesionales, sino además con escenarios sociales con el fin de materializar los desafíos que en la actualidad se imponen a la Universidad" (Mendoza, 2016).

Es evidentemente que las funciones misionales de Extensión Universitaria, Investigación y Docencia están totalmente ligadas con lo social, éste es parte integral de cada una de ellas. Las "actividades que se orientan al desarrollo físico, psico-afectivo, espiritual y social de los estudiantes, docentes y personal administrativo, así como los recursos humanos, infraestructura e inversión, dedicados a este proceso y población beneficiada" (Sistema Nacional de Información de la Educación Superior-SNIES, Ministerio de Educación Nacional-MEN, 2015) 
No obstante, la Extensión a diferencia de la Docencia e Investigación, se definió en la Ley 80 con el Artículo 160 promulga que: "como parte de la misión de las IES, enfocada en actividades de extensión científico-cultural y de servicio a la comunidad. Las actividades científicas y culturales están destinadas a la difusión de los conocimientos; y las actividades de servicio son aquellas que primordialmente buscan satisfacer necesidades específicas e inmediatas de la sociedad" (Decreto Ley 80, 1980).

A título ilustrativo se norma con la Ley 30 (1992, Art. 120) que: "La extensión comprende los programas de educación permanente, cursos, seminarios y demás programas destinados a la difusión de los conocimientos, al intercambio de experiencias, así como las actividades de servicio tendientes a procurar el bienestar general de la comunidad y la satisfacción de las necesidades de la sociedad" so pretexto de articular las IES a la realidad del país, en tal sentido considera la tercera función misional como Proyección Social o Extensión.

Para el caso de la universidad en cuestión, con base en la Ley 30 (1992, Art. 120), el Consejo Superior de la Institución, aprobó un Modelo Institucional de Acreditación, direccionado a la función sustantiva de Proyección Social. Para tal efecto, y con el fin de dar fuerza a la estructura orgánica de la Oficina de Proyección Social como asesora de Rectoría, y promulgó la Proyección Social (Extensión) junto con las otras funciones, (...) suprimiendo las Divisiones de Investigaciones, Extensión y Apoyo Académico, para crearlas a nivel directivo con el propósito de permitirles una dirección más apropiada a la naturaleza de las funciones que deben desarrollar a estas dos áreas misionales (Unicolmayor, Acuerdo 08, 2000).

Si bien es cierto, el Artículo Segundo del mismo Acuerdo, determinó que las funciones del Artículo 11 (Unicolmayor, Acuerdo 012, 2000) la División de Extensión y Apoyo Académico, fueran asumidas por la Vicerrectoría Académica, con el Acuerdo 005 (Unicolmayor, 1998). Se entiende entonces que la Extensión quedó incursa en la función misional de docencia a través de la Vicerrectoría Académica. Con base en la Autonomía Universitaria que permite a las IES organizar su estructura administrativa; y en este caso la División de Extensión y Apoyo Académico se disipa, se eclipsa y desaparece.

\section{RESUltados y DiscusióN}

Inicialmente se condujo la investigación en términos teóricos, abordando la temática de la consolidación del movimiento extensionista a nivel mundial. Donde se puede evidenciar que la Extensión Universitaria responde a un modelo de integración de la universidad con la sociedad, aplicada de acuerdo con las necesidades socioeconómicas de cada región o país.

Por otra parte, la concepción de universidad social nace del modelo de Responsabilidad Social que es totalmente al sector empresarial y los empleados, para luego rediseñada en el sector de la educación en términos de Responsabilidad Social Universitaria y finalmente adoptada como Proyección Social para las IES.

Para el caso colombiano, no hay una línea que caracterice uniformidad en el nombre de la tercera función misional en las IES públicas del orden nacional. 
TABLA 1.

Tercera Función Misional en IES Públicas del Orden Nacional

\begin{tabular}{ll}
\hline \multicolumn{1}{c}{ Universidad } & \multicolumn{1}{c}{ Tercera Función } \\
\hline Universidad Abierta y a Distancia & Proyección Social \\
Universidad Colegio Mayor de Cundinamarca & Proyección Social \\
Universidad de Córdoba & Investigación y Extensión \\
Universidad de Caldas & Proyección Social \\
Universidad de la Amazonía & Investigación y Extensión \\
Universidad de los Llanos Orientales & Proyección Social \\
Universidad del Cauca & Proyección Social \\
Universidad del Pacífico & Proyección Social \\
Universidad Militar Nueva Granada & Proyección Social y Extensión \\
Universidad Nacional de Colombia & Extensión \\
Universidad Pedagógica Nacional & Gestión Universitaria y Extensión \\
Universidad Popular del Cesar & Proyección Social \\
Universidad Sur Colombiana & Proyección Social \\
Universidad Tecnológica de Colombia & Extensión \\
Universidad Tecnológica de Pereira & Extensión \\
Universidad Tecnológica del Choco & Proyección Social y Extensión \\
\hline
\end{tabular}

Fuente: https://www.universidad.edu.co

De este modo, las encuestas realizadas a la comunidad académico-administrativa de la universidad en cuestión, dan clara muestra que hay un total desconocimiento de la concepción y operacionalidad de Extensión Universitaria en calidad de la tercera función misional.

TABLA 2.

Preguntas de las Encuestas con respecto a Extensión en Unicolmayor

\begin{tabular}{l}
\hline \multicolumn{1}{c}{ Preguntas y nivel de conocimiento } \\
\hline Conoce cuál es el proceso de Extensión en la universidad? \\
Conoce cuales son las actividades de la sede de Extensión en la universidad? \\
Conoce el portafolio de servicios educativos de la sede de Extensión? \\
Conoce temas específicos de Extensión Universitaria? \\
Conoce en que cursos de Extensión puede participar?
\end{tabular}

Fuente: Elaboración propia.

En lo esencial, al sistematizar y graficar las encuestas de Estudiantes (E), Docentes (D), Administrativos (A), asumiendo el 95\% de confiabilidad de la muestra de Unicolmayor, el resultado no es el mejor, el Nivel de Conocimiento (NC), de la muestra, se ubica en el rango de poco y muy poco conocimiento, es decir en el nivel entre 1 y 2 para una escala de 1 a 5 . 
Se puede indicar falta de descernimiento y nula capacitación en Extensión Universitaria. En consecuencia, el desconocimiento sobre este tema, implica ofertarla y gestionarla de forma inadecuada por parte de la Oficina de Proyección social de esta universidad.

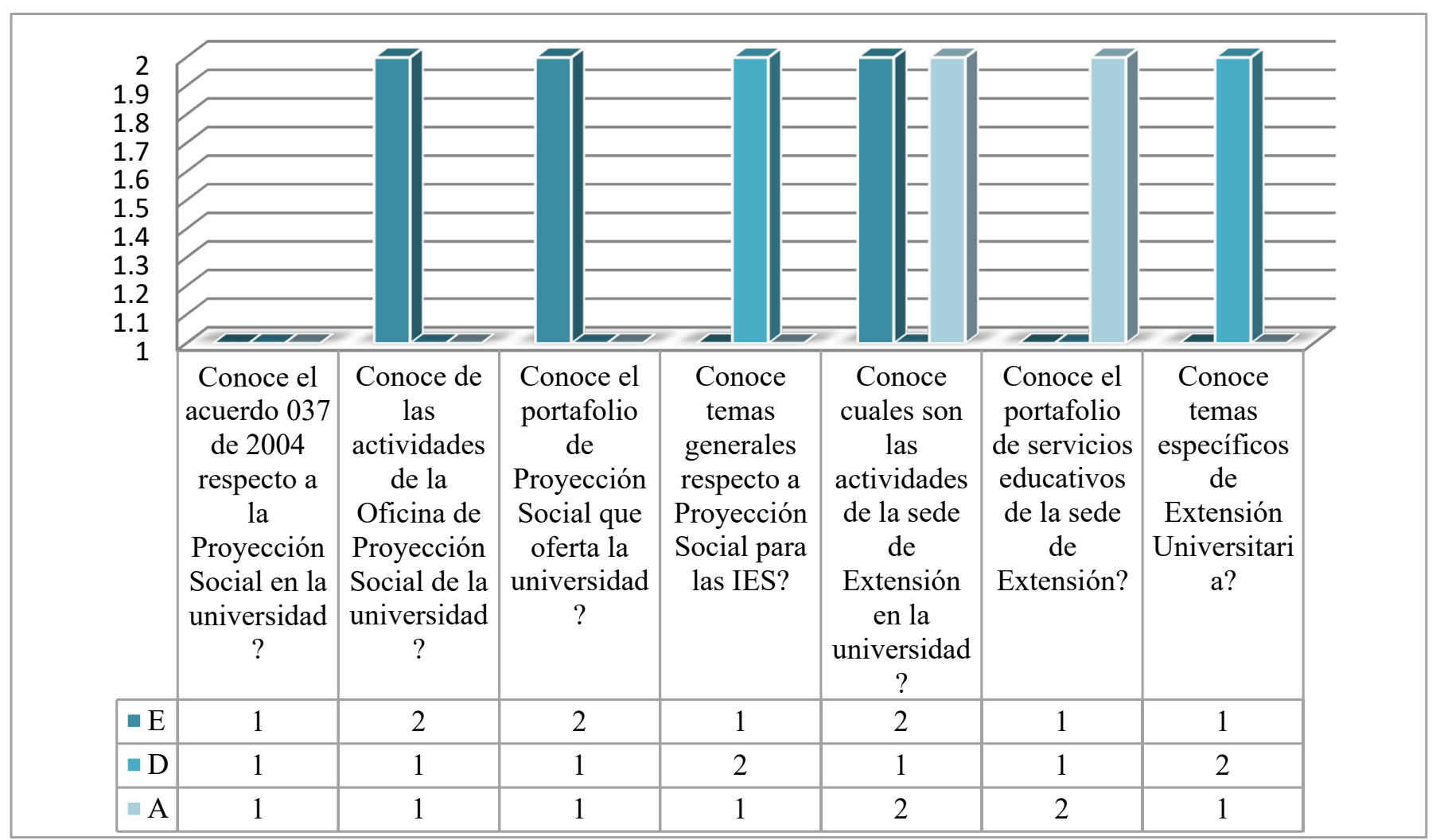

Figura 1. Nivel de Conocimiento con respecto a Extensión Universitaria Fuente: Elaboración propia.

Unicolmayor optó por Proyección Social como tercera función misional y sus estatutos consideran la Extensión, además posee una sede de Extensión, que por cierto no aparece en la estructura orgánica de la universidad, ofertando actividades de Extensión con cursos Extensión en Gastronomía, Belleza y Cosmetología, entre otros, propios más bien de unidad de bienestar social. Bajo las anteriores consideraciones la Extensión de la universidad no responde a los parámetros de la educación superior.

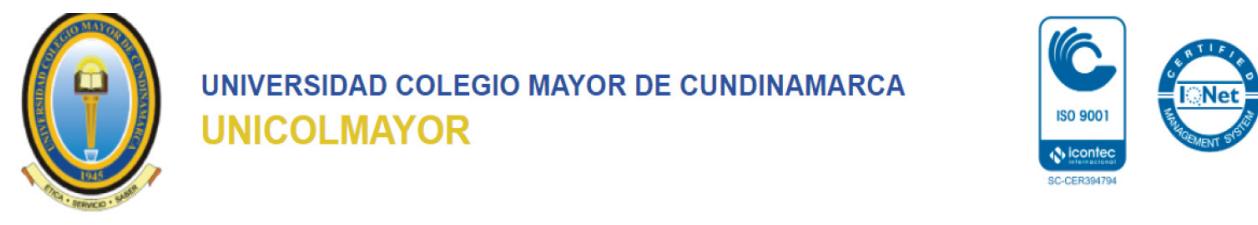

Cursos de Extensión

\begin{tabular}{|l|l|l|}
\hline ÁREA ARTES MANUALES & ÁREA DE BELLEZA Y COSMETOLOGÍA & ÁREA GASTRONOMÍA \\
\hline ÁREA DE CORTE Y CONFECCIÓN & ÁREA DE PINTURA & ÁREA LABORES \\
\hline ÁREA DE FORMACIÓN DE EMPRESAS & & \\
\hline
\end{tabular}

Figura 2. Cursos de Extensión en Unicolmayor

Fuente: Tomado de http://www.unicolmayor.edu.co/portal/index.php?idcategoria=209 
Analizados los resultados cuantitativos, éstos muestran la limitación conceptual con respecto a Extensión en términos de Extensión Universitaria y Proyección Social, factor que incurre en los procesos de formación académica. Se hace por tanto hincapié en la responsabilidad de desarrollar un concepto palpable y total de la Extensión como parte constitutiva de la Tercera Función Sustantiva en términos de Ley.

Finalmente, la Institución pertenece a la Red de Extensión Universitaria de ASCUN y su tercera función misional incluye Extensión (Acuerdo 08, 2007), pero como se evidencia, las actividades de Extensión ofertadas no son coherentes, ni están articuladas con las otras funciones misionales, tampoco con los programas académicos. Se detectó que la mayoría de actividades de Extensión académica, son propuestas y ejecutadas por las diferentes facultades académicas, según los informes y audiencias públicas de Rendición de Cuentas a la ciudadanía en diferentes periodos anual.

\section{CONCLUSiOnes}

Es evidente que el movimiento extensionista con más de dos siglos desde su origen en Europa y algo más de un siglo proclamada en Latinoamérica, ha demostrado que es una vía correcta en la interrelación comunidad-universidad, y como base para la tercera función misional en las IES.

Por consiguiente, Extensión Universitaria es una función integradora y aun cuando en la realidad no se le está dando la importancia de triada sino manejando de forma individual, consecuencia del desarrollo de la Extensión a niveles impropios en cuanto a su verdadera esencia para el lograr una interacción dinámica de las IES con la comunidad, por medio de la cual éstas hacen partícipe de su acervo humanístico y científico, a su vez recibe de la comunidad la realidad económica, social, orientación y estimulo.

Hay que repensar por qué de las dieciséis instituciones universitarias públicas del orden nacional se consideran modelos diferentes para la tercera función misional, y más aún que desconozcan la prospectiva del desarrollo institucional con modelos de tipo extensionista. En este sentido las expectativas en temas perentorios, nacientes y posibles respuestas a esquemas administrativos basados en la responsabilidad social y la proyección social que en están inmersos en la Extensión y que se han sugerido a las IES, pero la mayoría han desatendido los procesos de gestión extensionista, sin resultado en el binomio de las relaciones universidad-comunidad, dirigida de modo sistémico, eficiente, promocionado la cultura y capacitación para la sociedad intra y extra universitaria.

En última instancia, hay que diferenciar Proyección Social y Extensión Universitaria. La institución universitaria es una organización sin ánimo de lucro, no una empresa que se debate en la responsabilidad social empresarial, para que sus empleados tengan bienestar institucional en función de las utilidades y rendimientos financieros de los socios.

El papel de las IES no debe limitarse solo a la formación profesionalizante, basada en obtención de conocimientos, le incumbe la prospección de soluciones a los problemas del entorno. Es necesario salir de los muros universitarios e interactuar con el entorno, en la evolución de la ciencia y la tecnología a nivel mundial. Se debe acondicionar realmente el procedimiento de manejo administrativo, de tal manera que el desempeño de las instancias este en función de la académica y nuevos conocimientos. Es un reto arduo e impostergable si se desea superar el rezagamiento y descubrir opciones de desarrollo mayormente acordes con las exigencias del entorno, en un mundo globalizado. 


\section{REFERENCIAS}

Ardila, Y. (2011). Algunas implicaciones de la autofinanciación en la educación superior pública: el caso de la Universidad Nacional de Colombia. [Tesis Maestría]. Universidad Nacional de Colombia, Bogotá, D.C., Colombia. Disponible en http://bdigital.unal.edu. $\mathrm{co} / 3282 /$

Bowen, H. (1953). Social responsibilities of the businessman. New York: Harper \& Row.

CESU. (2014). Acuerdo por lo Superior 2034. Propuesta de política pública para la excelencia de la educación superior en Colombia en el escenario de la paz. Bogotá, D.C.: CESU. Disponible en https://dds.cepal.org/redesoc/publicacion?id=3723

Carlevaro, P. (2009). Universidad y Sociedad: proyección y vínculos. Reencuentro, 56(1), 40-51. Disponible en https://reencuentro.xoc.uam.mx/index.php/reencuentro/article/ view/717

Ferrero, A. (1999). Historia Crítica del Movimiento Estudiantil de Córdoba. Tomo I (19181943). Córdoba: Alción.

García-Perdiguero, T. (2003). La responsabilidad social de las empresas en un mundo global. Barcelona: Anagrama.

Hernández, R., Fernández, C. y Baptista, P. (2003). Metodología de la Investigación. México, D.F.: McGraw-Hill.

Khan, J. (1989). Design of a workshop to train P.S.U. faculty as international consultants in youth development (professional paper in Extension Education). Pennsylvania: The Pennsylvania State University.

Labrandero, M. y Santander, L. (1987). Extensión académica: una función del sistema universitario. Revista de la Educación Superior, (47), 1-10. Recuperado de http://publicaciones.anuies.mx/pdfs/revista/Revista47_S2A2ES.pdf

Lizcano, J. L. y Moneva, J. M. (2003). Marco conceptual de la responsabilidad social corporativa. [Documento AECA]. Madrid: Comisión de Responsabilidad Social Corporativa de AECA.

Martínez, M. (2006). La investigación cualitativa. Revista IIPSI, 9(1). 123-146. Recuperado de https://sisbib.unmsm.edu.pe/bvrevistas/investigacion_psicologia/v09_n1/pdf/ a09v9n1.pdf

Mendoza, C. (2016). Investigación en modo 3: Una alternativa para la articulación investigación e intervención en educación superior. Revista Guillermo de Ockham, 24(2), 103-110. Disponible en https://revistas.usb.edu.co/index.php/GuillermoOckham/article/view/2635

Misas, G. (2004). La educación superior en Colombia: análisis y estrategias para su desarrollo. Bogotá, D.C.: UNAM.

Morales, M., Mira, G. y Arias, M. (octubre, 2010). Enfoques y retos de la función de extensión universitaria como mecanismo de integración: Universidad, Empresa, Estado. En, UNAL, II Congreso Internacional de Gestión Tecnológica e Innovación, Bogotá, D. C., Colombia. Disponible en http://cogestec.ingenio.com.co/db/separated/2010\%20(79).pdf

Posada A. (1911). La extensión universitaria. Madrid: BILE.

Rábade, M. (1996). Las universidades en la Edad Media. Madrid. Arco/Libros (Cuadernos de Historia). Madrid: Arco Libros - La Muralla, S.L 
Ravelo, E. (2018). A cien años de la Reforma Universitaria de Córdoba. Hacia un nuevo manifiesto de la educación superior latinoamericana. ESS, 25(25), 183-196. Disponible en http://www.iesalc.unesco.org/ess/index.php/ess3/article/view/63

República de Colombia. Congreso de la República. (23 de enero de 2009). Por la cual se modifica la Ley 29 de 1990, se transforma a Colciencias en Departamento Administrativo, se fortalece el Sistema Nacional de Ciencia, Tecnología e Innovación en Colombia y se dictan otras disposiciones. [Ley 1286]. Diario Oficial No. 47.241. Disponible en https://minciencias.gov.co/node/302

República de Colombia. Congreso de la República. (28 de diciembre de 1992). Por el cual se organiza el servicio público de la Educación Superior en Colombia. [Ley 30]. Diario oficial No. 40.700. Disponible en https://minciencias.gov.co/node/269

República de Colombia. Congreso de la República. (22 de enero de 1980). Por el cual se organiza el sistema de educación postsecundaria. [Decreto 80]. Diario oficial No. 37.863. Recuperado de https://normograma.info/men/docs/pdf/decreto_0080_1980.pdf

República de Colombia. MEN. (5 de agosto de 2015). Por la cual se dictan disposiciones relacionadas con la administración de la información en el Sistema Nacional de Información de la Educación Superior — SNIES y se deroga la Resolución 1780 de 2010. [Resolución 12161]. Diario Oficial No. 50.043. Recuperado de https://snies.mineducacion.gov.co/1778/articles-391237_RESOLUCION_12161_DE_2015.pdf

Rosenberg, R. (1982). Beyond Separate Spheres: Intellectual Roots of Modern Feminism. New Haven: Yale University Press.

Serna, G. (2007). Misión social y modelos de extensión universitaria: del entusiasmo al desdén. Revista Iberoamericana de Educación, 43(3), 3-25. Disponible en https://rieoei.org/RIE/article/view/2324

Serna, G. (2004). Modelos de extensión universitaria en México. Revista Iberoamericana de Educación, 43(3), 1-7. Disponible en https://rieoei.org/historico/ deloslectores/1662Aquiles.pdf

Tünnermann, C. (2003). La universidad latinoamericana ante los retos del siglo XXI. México, D.F.: Colección UDUAL. Recuperado de www.udual.org/CIDU/CoIDUAL/tunner/ capitulo10.pdf

Tünnermann, C. (2000). El nuevo concepto de la extensión universitaria y difusión cultural y su relación con las políticas de desarrollo cultural en América Latina. Anuario de Estudios Centroamericanos, 4(1), 93-126. Disponible en https://revistas.ucr.ac.cr/ index.php/anuario/article/view/3305

UDUAL. (1972). II Conferencia Latinoamericana de Difusión Cultural y Extensión Universitaria. [Online]. Disponible en http://publicaciones.anuies.mx/pdfs/revista/Revista2_S3A1ES.pdf

UDUAL. (1957). Primera Conferencia Latinoamericana de Extensión Universitaria y Difusión Cultural. [Online]. Disponible en http://extension.fcien.edu.uy/wp-content/ uploads/2013/02/DefinicionExtension_ClaustroFCien_2010.pdf

UniColMayor. (5 de agosto de 2004). Reglamento de Proyección Social. [Acuerdo 037]. Recuperado de http://www.unicolmayor.edu.co/portal//recursos_user///Normatividad/Consejo\%20Academico/Acuerdos2004/Acuerdo037de2004.pdf 
UniColMayor. (10 de abril de 2000). Estructura Orgánica. [Acuerdo 012]. Recuperado de http://www.unicolmayor.edu.co/portal/index.php?idcategoria=2370

UniColMayor. (2 de marzo de 2000). Idoneidad estudiantes de derecho. [Acuerdo 008]. Recuperado de http://www.unicolmayor.edu.co/portal//index.php?idcategoria=18142

UnicolMayor. (9 de marzo de 1998). Actualización proyecto educativo. [Acuerdo 005]. Recuperado de http://www.unicolmayor.edu.co/portal/index.php?idcategoria=18207

UNAL. (20 de octubre de 2009). Acuerdo 036 de 2009 del Consejo Superior Universitario bajo el que se rige la Extensión Universidad Nacional de Colombia. [Online]. Recuperado de https://extension.unal.edu.co/fileadmin/recursos/extension/docs/normatividad/ acuerdo_036_2009.pdf

Vallaeys, F. (2006). Responsabilidad universitaria: hacia una definición madura del concepto. Lima: Universidad Católica del Perú.

Guillermo Tenorio Bohórquez es Doctor en Proyectos con énfasis en Investigación. Magister en Docencia, Especialista en Gerencia de Proyectos, Economista, Profesional Proyección Social. Miembro en Comités de: Currículo, Facultad, Evaluación de Currículos, Autoevaluación, Registro Calificado y Acreditación de Programas e Institucional, Jurado y Director de Proyectos de Grado, Docente Investigador. Docente a nivel postgrado y pregrado, Jefatura área económica, financiera y otras, Elaboración de Documentos Maestros para Nuevos Programas, manejo de plataforma Moodle, Teams, Zoom, Representaciones institucionales ante Afadeco y Aceun Ascun. https://orcid.org/0000-0003-3617-1090 